BRX-TH 478

\title{
(Dis)continuities of Massless Limits in Spin 3/2-mediated Interactions and Cosmological Supergravity
}

\author{
S. DESER AND A. WALDRON \\ Physics Department, Brandeis University, Waltham, MA 02454, USA \\ deser, wally@brandeis. edu
}

\begin{abstract}
We extend to its spin $3 / 2$ supersymmetric partner the very recent demonstration that the massless limit of massive spin 2 exchange amplitudes can be made continuous in background AdS spaces, in contrast to the known flat space discontinuities for both systems. In an AdS background, unlike spin 2 where the limit $m \rightarrow 0$ is the massless one, spin $3 / 2$ "masslessness" requires $m \rightarrow \sqrt{-\Lambda / 3}$, the supergravity value tuning the mass and cosmological constant that uniquely provides gauge invariance and two helicities. We find that continuity of the spin 3/2-mediated exchange amplitude can be regained in two "massless" limits $m \rightarrow 0$ and $m \rightarrow \sqrt{-\Lambda / 3}$; only the latter corresponds to cosmological supergravity.
\end{abstract}




\section{Introduction}

There is an old, but still surprising, discontinuity in the interaction generated by spin 2 exchange between external, conserved sources $T_{\mu \nu}(x)$ in flat space: the massless limit yields a finitely different amplitude from the strictly massless case. This effect, first observed in [1], can be summarized by the following simple formula:

$$
\begin{gathered}
\mathcal{A}=G_{\alpha} \int d^{4} x\left(T^{\mu \nu} D T_{\mu \nu}^{\prime}-\alpha T_{\mu}^{\mu} D T^{\prime \nu}{ }_{\nu}\right), \\
m \equiv 0 \Rightarrow \alpha=\frac{1}{2} ; \quad m \rightarrow 0 \Rightarrow \alpha=\frac{1}{3},
\end{gathered}
$$

where $D$ is the scalar propagator and $G_{\alpha}$ the corresponding gravitational constants. If the latter are chosen to fit the observed Newtonian strength, i.e., in the non-relativistic cases where only the energy densities $T_{00}$ fail to vanish, then the corresponding light bendings $\left(T^{\prime \nu}{ }_{\nu}=0\right)$ are discretely different by $25 \%$, thereby observationally excluding any non-zero $m$, however minuscule $\rrbracket$.

It was then shown in [3] that, as one would expect from supersymmetry, there is a precisely parallel effect for a spin $3 / 2$ field coupled to a conserved fermionic source $j^{\mu}$. Specifically, the analogue of (四) is

$$
\begin{aligned}
\mathcal{A} & =g_{\alpha} \int d^{4} x\left(\bar{j}^{\mu} S j_{\mu}-\alpha \bar{j}^{\mu} S \gamma_{\mu} \gamma_{\nu} j^{\nu}\right), \\
m & \equiv 0 \Rightarrow \alpha=\frac{1}{2} ; \quad m \rightarrow 0 \Rightarrow \alpha=\frac{1}{3},
\end{aligned}
$$

where $S$ is the spin $1 / 2$ propagator, so the "dictionary" reads $T_{\mu \nu} \rightarrow j_{\mu}$, $T_{\mu}^{\mu} \rightarrow \gamma \cdot j$ and $D \rightarrow S$.

Very recently, the entire question has been revived [4, 5] for spin 2 with the unexpected result that continuity can in fact be recovered by taking the background to be AdS rather Minkowski. More precisely, it was found that the amplitude (11) becomes

$$
\mathcal{A}=G \int d^{4} x\left(T^{\mu \nu} D T_{\mu \nu}^{\prime}-\frac{a+1}{2 a+3} T_{\mu}^{\mu} D T^{\prime \nu}{ }_{\nu}\right)
$$

where $\Lambda=-a m^{2} \leq 0$. Here $\Lambda$ is the (negative) cosmological constant. Three "massless" limits are to be distinguished: (1) The previous Minkowski one at

\footnotetext{
${ }^{1}$ This conclusion holds at the linearized level. The generalization of massive spin 2 to a non-linear Einstein-like form is, however, problematical [2].
} 
$a=0$, where one sets $\Lambda=0$ first $(\Rightarrow \alpha=1 / 3$ ), with the consequent $m \rightarrow 0$ jump compared with the strictly massless case. (2) A "cosmological limit" $a \rightarrow \infty$, i.e. $m$ vanishing faster than $\Lambda$, which yields the welcome continuous

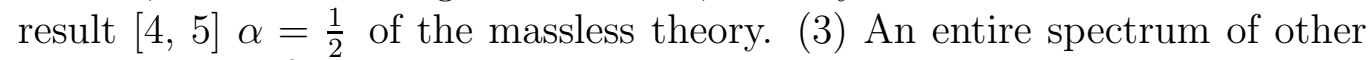
paths to the $m^{2}=0=\Lambda$ origin yielding any desired value of $\alpha$ in the range $1 / 3 \leq \alpha \leq 1 / 2$.

Although we seem to have replaced discontinuity by ambiguity, the correct physical choice is $m$ vanishing first, for which the model limits to the linearization of Einstein gravity in the presence of $\Lambda$, a perfectly gauge invariant system with just two excitations (and not 5 or 6 , as in the problematic non-linear extensions [2]). The final limit $\Lambda \rightarrow 0$ is therefore problem-free.

The results of [4, [5], themselves motivated by recent "brane-world" developments [7], beg for a parallel extension of the original flat space system [3] to AdS. We will indeed find the same physical conclusion, as befits the fact that $\mathcal{N}=1, D=4$ cosmological supergravity [8] describes a gauge invariant $s=3 / 2$ excitation with two degrees of freedom, just like its graviton partner [9]. There is, however, an amusing new wrinkle here, related to the fact that the "massless" gravitino, unlike the graviton, in fact does have a "mass term". (Neither propagate on the null cone in AdS spaces, however [10].) Consequently, the correct limit that yields the continuous result is not setting the parameter $m \rightarrow 0$ first but rather letting

$$
m \rightarrow \sqrt{-\Lambda / 3},
$$

the "tuned" gauge invariant (i.e. locally supersymmetric) value which we will review later in the text. Our result is that the exchange amplitude connects smoothly to the strictly massless flat case when one first takes the massless cosmological supergravity limit (4) and thereafter $\Lambda \rightarrow 0$. Interestingly enough, we also find that the limit $m \rightarrow 0$, followed by $\Lambda \rightarrow 0$ can be made to connect smoothly to the massless amplitude ${ }^{\text {s. }}$. It is tempting to view this bifurcation of possible limits for spin $3 / 2$ as AdS and dS phenomena, respectively (recall that the spin 2 result of [由, [5] is valid for both spacesit)

\footnotetext{
${ }^{2}$ Recently a study of massive spin 2 in general gravitational backgrounds has been carried out with the result that the correct excitation count holds for backgrounds where the traceless Ricci tensor vanishes [6].

${ }^{3}$ As this work was being completed, reference [11] appeared. There the $m \rightarrow 0$ nongauge invariant "de Sitter" limit is presented and agrees with our result.

${ }^{4}$ For the spin 2 dS case, see 12].
} 
although the absence of a dS supergravity theory [13] makes a precise relation problematic. However, as we shall see, the current conservation law necessary to derive the "de Sitter" result is not the usual supergravity one for spin $3 / 2$ fields in AdS space. Further, for masses $m>\sqrt{-\Lambda / 3}$, it is not possible to connect to the the $m \rightarrow 0$ regime without passing through the massless point $m=\sqrt{-\Lambda / 3}$. We conclude, therefore, that the physical AdS massless limit $m \rightarrow \sqrt{-\Lambda / 3}$ uniquely resolves the spin $3 / 2$ supergravity analogue of the van Dam-Veltman-Zhakarov discontinuity.

\section{Spin $3 / 2$}

The massive spin 3/2 Lagrangian coupled to gravity is

$$
\mathcal{L}=-\sqrt{-g} \bar{\psi}_{\mu} \gamma^{\mu \nu \rho} \mathcal{D}_{\nu} \psi_{\rho}
$$

where the mass term has been introduced by defining

$$
\mathcal{D}_{\mu} \equiv D_{\mu}+\frac{m}{2} \gamma_{\mu}
$$

satisfying $\left[\mathcal{D}_{\mu}, \mathcal{D}_{\nu}\right]=\left[D_{\mu}, D_{\nu}\right]+\left(m^{2} / 2\right) \gamma_{\mu \nu}$. The covariant derivative on the Rarita-Schwinger field reads

$$
D_{\mu} \psi_{\nu}=\partial_{\mu} \psi_{\nu}-\Gamma_{\mu \nu}^{\rho} \psi_{\rho}+\frac{1}{4} \omega_{\mu m n} \gamma^{m n} \psi_{\nu}
$$

with commutator

$$
\left[D_{\mu}, D_{\nu}\right] \psi_{\rho}=-R_{\mu \nu \rho}^{\sigma}(g) \psi_{\sigma}+\frac{1}{4} R_{\mu \nu m n}(\omega) \gamma^{m n} \psi_{\rho}
$$

We generally drop the labels $g$ and $\omega$ with the curvature convention $R_{\mu \nu \rho \sigma} \equiv$ $R_{\mu \nu \rho \sigma}(g)=-e_{\rho}^{a} e_{\sigma}^{b} R_{\mu \nu a b}(\omega)$. The Rarita-Schwinger field equation is

$$
\gamma^{\mu \nu \rho} \mathcal{D}_{\nu} \psi_{\rho}=0
$$

In an AdS background, we have

$$
R_{\mu \nu \rho \sigma}=-\frac{2 \Lambda}{3} g_{\mu[\rho} g_{\sigma] \nu}, \quad\left[D_{\mu}, D_{\nu}\right] \psi_{\rho}=\frac{2 \Lambda}{3} g_{\rho[\mu} \psi_{\nu]}+\frac{\Lambda}{6} \gamma_{\mu \nu} \psi_{\rho} .
$$

\footnotetext{
${ }^{5}$ Our metric is "mostly plus" and Dirac matrices are in turn "mostly hermitean". We (anti)symmetrize with unit weight; for details see [14].
} 
Computations are vastly simplified by introducing half-integer spin Lichnerowicz operators [15]

$$
\begin{aligned}
\mathcal{D}_{L}^{3 / 2} \psi_{\mu} & \equiv \gamma_{\mu \nu \rho} D^{\nu} \psi^{\rho}+\not D \psi^{\mu}=2 \not D \psi_{\mu}+\gamma_{\mu}(\not D \gamma \cdot \psi-D . \psi)-D_{\mu} \gamma \cdot \psi \\
\mathcal{D}_{L}^{1 / 2} \chi & \equiv \not D \chi
\end{aligned}
$$

with the useful properties

$$
\begin{aligned}
\mathcal{D}_{L}^{3 / 2} \gamma_{\mu} \chi & =\gamma_{\mu} \mathcal{D}_{L}^{1 / 2} \chi \\
\mathcal{D}_{L}^{3 / 2} D_{\mu} \chi & =D_{\mu} \mathcal{D}_{L}^{1 / 2} \chi \\
\left(\mathcal{D}_{L}^{1 / 2}\right)^{2} \chi & =\left(D^{2}-\Lambda\right) \chi
\end{aligned}
$$

The final ingredient we need is the covariant transverse-gamma-traceless decomposition in AdS:

$$
\begin{aligned}
\psi_{\mu} & =\psi_{\mu}^{\mathrm{TT}}+\gamma_{\mu} \frac{1}{3 D^{2}+\Lambda}\left(D^{2} \gamma \cdot \psi-\not D D \cdot \psi\right)-D_{\mu} \frac{1}{3 D^{2}+\Lambda}(\not D \gamma \cdot \psi-4 D \cdot \psi) \\
& \equiv \psi_{\mu}^{\mathrm{TT}}+\gamma_{\mu} \phi+D_{\mu} \chi, \quad D \cdot \psi^{\mathrm{TT}}=0=\gamma \cdot \psi^{\mathrm{TT}} .
\end{aligned}
$$

We will present two computations. The first treats the limit where the parameter $m \rightarrow 0$ in a cosmological space. As stated earlier, it does not correspond to the massless AdS supergravity limit but is, in some sense, the direct analogue of the spin 2 computation of [4, 5]. Recalling that the latter was valid in either dS or AdS space, for lack of a better name, we refer to this limit as the "de Sitter" calculation. The second computation takes the limit $m \rightarrow \sqrt{-\Lambda / 3}$, the massless locally supersymmetric point, and will verify the continuity of cosmological AdS supergravity.

We begin with the "de Sitter" case. Our method follows the lines of [5]. We compute a single particle exchange amplitude between two covariantly conserved spinor-vector sources $j_{\mu}$ and $\bar{j}_{\mu}, D \cdot j=0=\bar{j} \cdot \overleftarrow{D}$

$$
\mathcal{A}=\int d^{4} x \sqrt{-g} \bar{j}^{\mu} \Delta_{\mu \nu}^{3 / 2}(\Lambda) j^{\nu}=\int d^{4} x \sqrt{-g} \bar{j}^{\mu} \psi_{\mu}(j)
$$

where $\Delta_{\mu \nu}^{3 / 2}(\Lambda)$ is the propagator for a massive spin $3 / 2$ particle in an AdS space and the second equality is achieved by substituting the solution $\psi_{\mu}(j)$ of the Rarita-Schwinger equation with a source

$$
\gamma^{\mu \nu \rho} \mathcal{D}_{\nu} \psi_{\rho}=j^{\mu}
$$


Since the source $\bar{j}_{\mu}$ is covariantly conserved we only need to calculate $\psi_{\mu}^{\mathrm{TT}}(j)$ and $\phi(j)$. The second of these is computed as follows: Note that

$$
\begin{aligned}
\mathcal{D} . j & =\frac{1}{2} m \gamma \cdot j=\mathcal{D}_{\mu} \gamma^{\mu \nu \rho} \mathcal{D}_{\nu} \psi_{\rho}=-\frac{1}{2}\left(\Lambda+3 m^{2}\right) \gamma \cdot \psi \\
\gamma \cdot j & =(2 \not D-3 m) \gamma \cdot \psi-2 D \cdot \psi
\end{aligned}
$$

Solving and substituting these into (16) gives (note that $\left[\not D, D^{2}\right] \gamma \cdot j=0$ )

$$
\phi(j)=\frac{\Lambda(\not D-2 m)}{2\left(3 D^{2}+\Lambda\right)\left(\Lambda+3 m^{2}\right)} \gamma \cdot j .
$$

Proceeding to the computation of $\psi_{\mu}^{\mathrm{TT}}(j)$ we rewrite (18) in terms of the Lichnerowicz operator

$$
\left(\mathcal{D}_{L}^{3 / 2}-\not D\right) \psi_{\mu}-m \gamma_{\mu \nu} \psi^{\nu}=j_{\mu}
$$

Using $\left(\gamma_{\mu} \phi\right)^{\mathrm{TT}}=0=\left(D_{\mu} \chi\right)^{\mathrm{TT}}$ along with the properties of the Lichnerowicz operators produces the identities

$$
\left(\mathcal{D}_{L}^{3 / 2} \psi_{\mu}\right)^{\mathrm{TT}}=\mathcal{D}_{L}^{3 / 2} \psi_{\mu}^{\mathrm{TT}}=\left(2 \not D \psi_{\mu}\right)^{\mathrm{TT}}=2 \not D \psi_{\mu}^{\mathrm{TT}}, \quad\left(m \gamma_{\mu \nu} \psi^{\nu}\right)^{\mathrm{TT}}=m \psi_{\mu}^{\mathrm{TT}}
$$

Hence

$$
\psi_{\mu}^{\mathrm{TT}}(j)=\frac{1}{\frac{1}{2} \mathcal{D}_{L}^{3 / 2}+m} j_{\mu}^{\mathrm{TT}}=\frac{1}{\not D+m} j_{\mu}^{\mathrm{TT}} .
$$

For a covariantly conserved quantity, the transverse-gamma-traceless decomposition simplifies;

$$
j_{\mu}^{\mathrm{TT}}=j_{\mu}-\left(\gamma_{\mu} D^{2}-D_{\mu} \not D\right) \frac{1}{3 D^{2}+\Lambda} \gamma \cdot j .
$$

Using this result for $\bar{j}_{\mu}$ and equations (24) and (21) we obtain our result for the exchange amplitude ${ }^{6}$

$$
\begin{aligned}
& \mathcal{A}=\int d^{4} x \sqrt{-g}\left(\bar{j}^{\mathrm{TT}} \mu \frac{1}{\not D+m} j_{\mu}^{\mathrm{TT}}+\bar{j} \cdot \gamma \phi(j)\right) \\
& { }^{6} \text { The AdS identity } \\
& \frac{1}{\not D+m}\left(\gamma_{\mu} D^{2}-D_{\mu} \not D\right) \chi=-\left(\gamma_{\mu} D^{2}-D_{\mu} \not D\right) \frac{\not D+m}{D^{2}-m^{2}} \chi-\frac{\Lambda}{2}\left(\gamma_{\mu} \not D-4 D_{\mu}\right) \frac{1}{D^{2}-m^{2}} \chi
\end{aligned}
$$

for any spinor $\chi$ has also been used to derive this result. It follows from the identities $\not D^{2} \gamma_{\mu} \chi=\gamma_{\mu} D^{2} \chi$ and $\not D^{2} D_{\mu} \chi=D_{\mu} D^{2} \chi$. 


$$
\begin{aligned}
=\int d^{4} x & \sqrt{-g}\left(\bar{j}^{\mu} \frac{1}{\not D+m} j_{\mu}\right. \\
& \left.+\frac{1}{2} \bar{j} \cdot \gamma \frac{1}{3 D^{2}+\Lambda}\left[\frac{2 D^{2}(\not D+m)+\Lambda \not D}{\left(D^{2}-m^{2}\right)}+\frac{\Lambda(\not D-2 m)}{\left(\Lambda+3 m^{2}\right)}\right] \gamma \cdot j\right) .
\end{aligned}
$$

Firstly note that the apparent pole at $D^{2}=-\Lambda / 3$ is spurious; a simple calculation reveals a vanishing residue there. At the physical pole $D^{2}=m^{2}$ we find in the $\gamma \cdot j$ sector

$$
\mathcal{A}(\gamma \cdot j) \stackrel{D^{2} \rightarrow m^{2}}{\sim} \int d^{4} x \sqrt{-g} \bar{j} \cdot \gamma \frac{\left(m^{2}+\frac{1}{2} \Lambda\right) \not D+m^{3}}{3 m^{2}+\Lambda} \frac{1}{D^{2}-m^{2}} \gamma \cdot j
$$

In the limit where we first go to flat space and thereafter take the mass to zero, we get

$$
\mathcal{A}(\gamma \cdot j) \sim \frac{1}{3} \int d^{4} x \bar{j} \cdot \gamma \frac{1}{\not} \gamma \cdot j, \quad(\Lambda \rightarrow 0 \text { then } m \rightarrow 0) .
$$

For continuity, the prefactor should be $1 / 2$ and this is the spin $3 / 2$ version [3] of the famous spin 2 discontinuity. Now take the limit $m \rightarrow 0$ first

$$
\mathcal{A}(\gamma \cdot j) \sim \frac{1}{2} \int d^{4} x \bar{j} \cdot \gamma \frac{1}{\not \partial} \gamma \cdot j, \quad(m \rightarrow 0 \text { then } \Lambda \rightarrow 0) .
$$

The prefactor is now $1 / 2$ and therefore connects smoothly to the massless flat space result]

$$
\mathcal{A}=\int d^{4} x \bar{j}^{\mu}\left(\frac{\eta_{\mu \nu}}{\not \partial}+\gamma_{\mu} \frac{1}{2 \not \partial} \gamma_{\nu}\right) j^{\nu}, \quad(m \equiv 0) \text {. }
$$

As claimed in the introduction, the "de Sitter" limit is continuous.

We now turn to the AdS case and ask the question: When is a spin 3/2 particle massless in an AdS space? The answer [9] is not at the value of the parameter $m=0$. Rather, masslessness occurs whenever a gauge invariance appears. The relevant gauge invariance for spin 3/2 in AdS is under transformations

$$
\delta \psi_{\mu}=\mathcal{D}_{\mu} \epsilon=\left(D_{\mu}+\frac{1}{2} m \gamma_{\mu}\right) \epsilon, \text { with } m^{2}=-\Lambda / 3
$$

\footnotetext{
${ }^{7}$ See [3], a pedagogical derivation may be found in [16].
} 
i.e., at the point where the model becomes (linearized) cosmological supergravity [9]. This is obvious from the constraint (19) which is now the Bianchi identity of the locally supersymmetric gauge theory

$$
\mathcal{D}_{\mu} \gamma^{\mu \nu \rho} \mathcal{D}_{\nu} \psi_{\rho}=0, \text { with } m^{2}=-\Lambda / 3 .
$$

Further the "de Sitter" calculation presented above is singular in this limit due to the final term in (26). The reason is simply that we took $D . j=0$, which is not consistent with the massless Bianchi identity (32).

The remedy is to impose the conservation law of the underlying massless theory for the current $J_{\mu}$

$$
D . J+\frac{1}{2} m\left(\frac{\Lambda}{-3 m^{2}}\right)^{\beta} \gamma \cdot J=0
$$

We have included the parameter $\beta$ since any of the above choices reduces to the conservation law $\mathcal{D} . j=0$ of the massless theory at $3 m^{2}=-\Lambda$. However, let us now argue that the choice $\beta=1 / 2$ is the correct one physically. In that case

$$
D . J+\frac{1}{2} \sqrt{-\Lambda / 3} \gamma . J=0
$$

and the external current obeys the conservation law of the massless theory for any massive deformation $m$; we hold the background cosmological spacetime constant and study the limit $m \rightarrow \sqrt{-\Lambda / 3}$. Indeed, an external current cannot depend on the particular dynamical theory to which it couples, but can, of course, involve the external background geometry. In other words, equation (34) is the analogue of covariant conservation in AdS space for spin $3 / 2$ fields.

A simple example is convincing: Consider the flat space massive spin $3 / 2$ propagator

$S_{\mu \nu}^{F}=\frac{1}{\partial^{2}-m^{2}}\left[\left(\eta_{\mu \nu}-\frac{\partial_{\mu} \partial_{\nu}}{m^{2}}\right)(\not \partial-m)+\frac{1}{3}\left(\frac{\partial_{\mu}}{m}-\gamma_{\mu}\right)(\not \partial+m)\left(\frac{\partial_{\nu}}{m}-\gamma_{\nu}\right)\right]$,

and take its expectation between currents satisfying $\partial \cdot \mathcal{J}+\frac{1}{2} m \gamma \cdot \mathcal{J}=0$. The result is

$$
\overline{\mathcal{J}}^{\mu} S_{\mu \nu}^{F} \mathcal{J}^{\nu}=\overline{\mathcal{J}}^{\mu} \frac{1}{\not \partial+m} \mathcal{J}_{\mu}+\frac{1}{2} \overline{\mathcal{J}} \cdot \gamma \frac{\not \partial+2 m}{\partial^{2}-m^{2}} \gamma \cdot \mathcal{J} .
$$

This amplitude connects smoothly with the massless result as $m \rightarrow 0$ since the coefficient of the gamma-trace terms is $1 / 2$. This does not mean that 
there is no Minkowski discontinuity however, since here the current $\mathcal{J}_{\mu}$ (which transforms under variations of $m$ ), is not the conserved one of the underlying massless theory. (We note in passing, that although the calculation of the exchange amplitude for the choice $\beta=0$ in (33) is rather simple, the result yields no discontinuity since it is the cosmological analogue of (36)).

Our task now is to compute the exchange amplitude for currents obeying (34) and mass-parameter detuned from the supersymmetric value $3 m^{2}=$ $-\Lambda$. Defining

$$
\bar{m} \equiv \sqrt{-\Lambda / 3}
$$

we therefore eliminate D.J throughout via the relation (34),

$$
D . J=-\frac{1}{2} \bar{m} \gamma . J .
$$

The method follows the above "de Sitter" calculation. Firstly the gammatrace and $\mathcal{D}_{\mu}$-divergence of the Rarita-Schwinger equation

$$
\gamma^{\mu \nu \rho} \mathcal{D}_{\nu} \psi_{\rho}=J^{\mu}
$$

yield

$$
\gamma \cdot \psi=-\frac{\gamma \cdot J}{3(m+\bar{m})}, \quad D \cdot \psi=-\frac{2 \not D+3 \bar{m}}{m+\bar{m}} \gamma . J
$$

(We stress that $\bar{m} \neq m$ has been used to derive the above.) In turn, the trace and transverse components of the field $\psi_{\mu}(J)$ are

$$
\phi(J)=\frac{\bar{m}(\not D+2 \bar{m})}{6(m+\bar{m})\left(D^{2}-\bar{m}^{2}\right)} \gamma . J, \quad \chi(J)=-\frac{\not D+2 \bar{m}}{3(m+\bar{m})\left(D^{2}-\bar{m}^{2}\right)} \gamma . J
$$

Again, the half-integer Lichnerowicz identities (23) imply that a one-particle exchange amplitude takes the form

$$
\mathcal{A}=\int d^{4} x \sqrt{-g}\left(\bar{J}^{\mathrm{TT} \mu} \frac{1}{\not D+m} J_{\mu}^{\mathrm{TT}}+\bar{J} \cdot \gamma\left[\phi(J)-\frac{1}{2} \bar{m} \chi(J)\right]\right) .
$$

where we have applied (38) to eliminate $\bar{J} . \overleftarrow{D}$. Finally, the transversegamma-traceless decomposition simplifies by virtue of (38)

$$
J_{\mu}^{\mathrm{TT}}=J_{\mu}-\left[\left(\gamma_{\mu} D^{2}-D_{\mu} \not D\right)+\frac{1}{2} \bar{m}\left(\gamma_{\mu} \not D-4 D_{\mu}\right)\right] \frac{1}{3 D^{2}+\Lambda} \gamma . J
$$


Inserting (43) and (41) into the amplitude (42) gives the central result

$$
\begin{aligned}
\mathcal{A} & =\int d^{4} x \sqrt{-g}\left(\bar{J}^{\mu} \frac{1}{\not D+m} J_{\mu}\right. \\
+ & \frac{1}{3} \bar{J} \cdot \gamma \frac{1}{D^{2}-\bar{m}^{2}}\left[\frac{D^{2}(\not D+m+\bar{m})-\bar{m}\left([2 \bar{m}-m] \not D+3 \bar{m}^{2}-m \bar{m}\right)}{\left(D^{2}-m^{2}\right)}\right. \\
& \left.\left.+\frac{\bar{m}(\not D+2 \bar{m})}{m+\bar{m}}\right] \gamma . J\right) \cdot
\end{aligned}
$$

Again an apparent pole at $D^{2}=\bar{m}^{2}$ is spurious: it is easy to verify that its residue cancels (a strong check of our algebra). Observe also that, unlike for $(26)$, there is no singularity at $m=\bar{m}=\sqrt{-\Lambda / 3}$. At the physical pole $D^{2}=m^{2}$ we find in the $\gamma . J$ sector

$$
\mathcal{A}(\gamma . J) \stackrel{D^{2} \rightarrow^{2}}{\sim} \int d^{4} x \sqrt{-g} \bar{J} \cdot \gamma \frac{(m+2 \bar{m}) \not D+m^{2}+2 m \bar{m}+3 \bar{m}^{2}}{3(m+\bar{m})\left(D^{2}-m^{2}\right)} \gamma . J
$$

Firstly, consider the limit in which we expect a discontinuity, namely setting the cosmological constant $\Lambda$ to zero first, and only thereafter the mass $m \rightarrow 0$,

$$
\mathcal{A}(\gamma \cdot J) \sim \frac{1}{3} \int d^{4} x \bar{J} \cdot \gamma \frac{1}{\not \partial} \gamma \cdot J, \quad(\Lambda \rightarrow 0 \text { then } m \rightarrow 0) .
$$

The prefactor should be $1 / 2$ and again we find the spin $3 / 2$ version of the spin 2 discontinuity.

Finally, we show that cosmological supergravity is free of any discontinuity. That means taking the (supersymmetric) limit

$$
m \rightarrow \sqrt{-\Lambda / 3} \Leftrightarrow m \rightarrow \bar{m}
$$

in which the theory truly becomes (AdS) massless. The result is

$$
\mathcal{A}(\gamma . J) \sim \frac{1}{2} \int d^{4} x \sqrt{-g} \bar{J} \cdot \gamma \frac{\not D+2 \bar{m}}{D^{2}-\bar{m}^{2}} \gamma . J
$$

\footnotetext{
${ }^{8}$ To check the details, the identity

$$
\frac{1}{\not D+m}\left(\gamma_{\mu} \not D-4 D_{\mu}\right) \chi=\left(\gamma_{\mu} \not D-4 D_{\mu}\right) \frac{\not D-m}{D^{2}-m^{2}} \chi-2\left(\gamma_{\mu} D^{2}-D_{\mu} \not D\right) \frac{1}{D^{2}-m^{2}} \chi
$$
}

valid for any spinor $\chi$, will be appreciated. 
This is the result for the exchange amplitude of cosmological supergravity. The final step is to take the flat space limit $\Lambda \rightarrow 0$ which, of course, is smooth

$$
\mathcal{A}(\gamma . J) \sim \frac{1}{2} \int d^{4} x \bar{J} \cdot \gamma \frac{1}{\not \partial} \gamma \cdot J, \quad(m \rightarrow \sqrt{-\Lambda / 3} \underline{\text { then }} \Lambda \rightarrow 0)
$$

The prefactor $1 / 2$ of course matches perfectly with the strictly massless result (30).

To summarize, we have seen that the spin $3 / 2$ one-particle exchange amplitude in AdS connects smoothly to (massless) cosmological supergravity. This requires the limit $m \rightarrow \sqrt{-\Lambda / 3}$ along with the current conservation law $\mathcal{D}(\Lambda) . J=0$ of the underlying massless supergravity theory. The flat massless Minkowski limit can be achieved continuously thereafter by taking $\Lambda \rightarrow 0$.

\section{Acknowledgments}

This work was supported by the National Science Foundation under grant PHY99-73935.

\section{References}

[1] H. van Dam and M. Veltman, Nucl. Phys. B22, 397 (1970); V. I. Zakharov, JETP Lett. 12, 312 (1970); L. D. Faddeev and A. A. Slavnov, Theor. Math. Phys. 3, 18 (1970); S. K. Wong, Phys. Rev. D3, 945 (1971).

[2] D. G. Boulware and S. Deser, Phys. Rev. D6, 3368 (1972).

[3] S. Deser, J. H. Kay and K. S. Stelle, Phys. Rev. D16, 2448 (1977).

[4] I. I. Kogan, S. Mouslopoulos and A. Papazoglou, "The $\mathrm{m} \rightarrow 0$ limit for massive graviton in $\mathrm{dS}(4)$ and $\operatorname{AdS}(4)$ : How to circumvent the van Dam-Veltman-Zakharov discontinuity," hep-th/0011138.

[5] M. Porrati, "No van Dam-Veltman-Zakharov discontinuity in AdS space," hep-th/0011152. 
[6] I. L. Buchbinder, D. M. Gitman, V. A. Krykhtin and V. D. Pershin, Nucl. Phys. B584, 615 (2000) hep-th/9910188.

[7] L. Randall and R. Sundrum, Phys. Rev. Lett. 83, 3370 (1999) hepph/9905221; Phys. Rev. Lett. 83, 4690 (1999) hep-th/9906064.

[8] P. K. Townsend, Phys. Rev. D15, 2802 (1977).

[9] S. Deser and B. Zumino, Phys. Rev. Lett. 38, 1433 (1977).

[10] S. Deser and R. I. Nepomechie, Phys. Lett. B132, 321 (1983); Annals Phys. 154, 396 (1984).

[11] P. A. Grassi and P. van Nieuwenhuizen, "No van Dam-VeltmanZakharov discontinuity for supergravity in AdS space," hep-th/0011278.

[12] A. Higuchi, Nucl. Phys. B282, 397 (1987); ibid. B325, 745 (1989).

[13] K. Pilch, P. van Nieuwenhuizen and M. F. Sohnius, Commun. Math. Phys. 98, 105 (1985).

[14] S. Deser, V. Pascalutsa and A. Waldron, Phys. Rev. D62, 105031 (2000) hep-th/0003011.

[15] A. Lichnerowicz, Institut des Hautes Études Scientifiques, 10, 293 (1961); Bull. Soc. Math. France, 92, 11 (1964).

[16] P. Van Nieuwenhuizen, Phys. Rept. 68, 189 (1981). 